\title{
Modular Elements of Geometric Lattices
}

\author{
RICHARD P. STANLEY
}

\section{Modular Elements}

Let $L$ be a finite geometric lattice of rank $n$ with rank function $r$. (For definitions, see e.g., [3, Chapter 2], [4], or [1, Chapter 4].) An element $x \in L$ is called a modular element if it forms a modular pair with every $y \in L$, i.e., if $a \leqslant y$ then $a \vee(x \wedge y)$ $=(a \vee x) \wedge y$. Recall that in an upper semimodular lattice (and thus in a geometric lattice) the relation of being a modular pair is symmetric; in fact $(x, y)$ is a modular pair if and only if $r(x)+r(y)=r(x \vee y)+r(x \wedge y)$ [1, p. 83]. Every point (atom) of a geometric lattice is a modular element. If every element of $L$ is modular, then $L$ is a modular lattice. The main object of this paper is to show that a modular element of $L$ induces a factorization of the characteristic polynomial of $L$. This is done in Section 2. First we discuss some other aspects of modular elements.

The following theorem provides a characterization of modular elements.

THEOREM 1. An element $x \in L$ is modular if and only if no two complements of $x$ are comparable.

Proof. If $x$ is modular and $x^{\prime}$ is a complement of $x$, then $r\left(x^{\prime}\right)=n-r(x)$. Hence all the complements of $x$ have the same rank and are incomparable.

Conversely, assume $x$ is not modular. Then there are elements $y<z$ such that $x \wedge y=x \wedge z$ and $x \vee y=x \vee z$. Let $p_{1}, p_{2}, \ldots, p_{n}$ be a basis for $L$ such that $p_{1}, p_{2}, \ldots, p_{s}$ is a basis for $x \wedge y ; p_{1}, p_{2}, \ldots, p_{t}$ is a basis for $y ; p_{1}, p_{2}, \ldots, p_{u}$ is a basis for $z$; and $p_{1}, p_{2}, \ldots, p_{v}$ is a basis for $x \vee y$. Thus $0 \leqslant s<t<u<v \leqslant n, r(x \wedge y)=s, r(y)=t, r(z)=u$, $r(x \vee y)=v$. Let

$$
\begin{aligned}
& x^{\prime}=p_{s+1} \vee p_{s+2} \vee \cdots \vee p_{t} \vee p_{v+1} \vee p_{v+2} \vee \cdots \vee p_{n} \\
& x^{\prime \prime}=x^{\prime} \vee p_{t+1} \vee p_{t+2} \vee \cdots \vee p_{u} .
\end{aligned}
$$

It is easily seen that $x^{\prime}$ and $x^{\prime \prime}$ are both complements of $x$ with $x^{\prime}<x^{\prime \prime}$.

In particular, an element $x$ with a unique complement $x^{\prime}$ is modular. For a stronger result, recall that an element $x$ is said to be in the center of an ordered set $P$ with 0 and 1 if $P=[0, x] \times\left[0, x^{\prime}\right]$ for some $x^{\prime}[1$, p. 67]. For an element $x$ to be in the center of a geometric lattice $L$, each of the following conditions is necessary and sufficient:

(1) $x$ is distributive (because $x$ is complemented, cf. $[1$, p. 69]), i.e., for any $y$, $z \in L$, the sublattice generated by $x, y, z$ is distributive.

(2) $x$ has a unique complement. This result appears to be new; Curtis Greene has Presented by G. Birkhoff. Received December 5, 1970. Accepted for publication in final form March 22, 1971 . 
in fact proved the more general result (unpublished) that the intersection (meet) of all the complements of any element of a geometric lattice $L$ is in the center of $L$.

(3) $x$ is a separator of $L[3$, Chapter 12], i.e., for any point $p$ and any copoint $q$ not containing $p$, either $p \leqslant x$ or $x \leqslant q$.

(4) $x$ is a standard element of $L[1$, p. 69] (this follows from (2) since a complemented standard element has a unique complement). The concept of standard elements is due to G. Grätzer.

\section{The Characteristic Polynomial}

The characteristic polynomial $p_{L}(\lambda)$ [4] of a geometric lattice $L$ is defined by

$$
p_{L}(\lambda)=\sum_{y \in L} \mu(0, y) \lambda^{n-r(y)},
$$

where $\mu$ denotes the Möbius function of $L$ (see [4]).

This polynomial was first considered by G. D. Birkhoff, while its connection with Möbius functions was noted by Garrett Birkhoff. If $x \in L$, the characteristic polynomial of the segment $[0, x]$ is denoted $p_{x}(\lambda)$.

The main result of this paper is the following factorization theorem: then

THEOREM 2. If $x$ is a modular element of a finite geometric latice $L$ of rank $n$,

$$
p_{L}(\lambda)=p_{x}(\lambda)\left[\sum_{b: x \wedge b=0} \mu(0, b) \lambda^{n-r(x)-r(b)}\right]
$$

The expression in brackets may be thought of as the characteristic polynomial of the order ideal $C(x)=\{b \mid x \wedge b=0\} . C(x)$ will have a 1 if and only if $x$ has a unique complement $x^{\prime}$ in $L$. In this case $C(x)=\left[0, x^{\prime}\right]$ and $L=[0, x] \times\left[0, x^{\prime}\right]$. Thus when $x$ has a unique complement Theorem 2 is trivial, since $p_{L_{1} \times L_{2}}=p_{L_{1}} p_{L_{2}}$.

To prove Theorem 2, we first prove two lemmas. The first lemma is a special case of some results of Schwan on modular pairs (see [1, Section IV.2]), but for the sake of completeness we include a proof. It is to be assumed throughout that $x$ is a modular element of the finite geometric lattice $L$, and that $L$ has rank $n$.

LEMMA 1. For any $a \in L$, the map

$$
\sigma_{a}:[a, a \vee x] \rightarrow[a \wedge x, x]
$$

defined by $\sigma_{a}(y)=x \wedge y$ is an isomorphism with inverse $\tau_{a}(y)=a \vee y$.

Proof. Clearly $\sigma_{a}$ and $\tau_{a}$ are order-preserving. By modularity of $x$, it is immediate that if $y \in[a \wedge x, x]$, then $\sigma_{a} \tau_{a}(y)=x \wedge(a \vee y)=(x \wedge a) \vee y=y$. Also if $y \in[a, a \vee x]$, then $\tau_{a} \sigma_{a}(y)=a \vee(x \wedge y)=(a \vee x) \wedge y=y$, and the proof follows. 
LEMMA 2. For any $y \in L, x \wedge y$ is a modular element of $[0, y]$.

Proof. Let $a \in[0, y]$ and let $b \leqslant a$. We need to show $b \vee((x \wedge y) \wedge a)=(b \vee(x \wedge y)) \wedge a$. Using the modularity of $x$, we have

$$
\begin{aligned}
(b \vee(x \wedge y)) \wedge a & =((b \vee x) \wedge y) \wedge a=(b \vee x) \wedge a=b \vee(x \wedge a) \\
& =b \vee(x \wedge(y \wedge a))=b \vee((x \wedge y) \wedge a) . \square
\end{aligned}
$$

Proof of Theorem 2. By Crapo's Complementation Theorem [2], if $a \in[0, y]$ then

$$
\mu(0, y)=\sum_{a^{\prime}, a^{\prime \prime}} \mu\left(0, a^{\prime}\right) \zeta\left(a^{\prime}, a^{\prime \prime}\right) \mu\left(a^{\prime \prime}, y\right),
$$

where $a^{\prime}$ and $a^{n}$ are complements of $a$ in $[0, y]$, and $\zeta$ is the zeta function of $[0, y]$. Choosing $a=x \wedge y$, then by Lemma 2 all the complements of $a$ have the same rank and hence are incomparable. Thus

$$
\mu(0, y)=\sum \mu(0, b) \mu(b, y),
$$

where the sum is over all complements $b$ of $x \wedge y$ in $[0, y]$, i.e., over all $b \in L$ satisfying $0 \leqslant b \leqslant y, b \wedge(x \wedge y)=0, b \vee(x \wedge y)=y$. Now $b \wedge(x \wedge y)=b \wedge x$, and by the modularity of $x, b \vee(x \wedge y)=(b \vee x) \wedge y$. Thus the sum (1) is over all $b \in L$ satisfying $b \wedge x=0$ and $y \in[b, b \vee x]$. Hence

$$
\begin{aligned}
p_{L}(\lambda) & =\sum_{y \in L} \mu(0, y) \lambda^{n-r(y)} \\
& =\sum_{y \in L} \sum_{b \wedge x=0} \mu(0, b) \mu(b, y) \lambda^{n-r(y)} \\
& =\sum_{b \wedge x=0} \sum_{y \in[b, b \vee b \times]} \mu(0, b) \mu(b, y) \lambda^{n-r(y)}
\end{aligned}
$$

Now by Lemma 1 , as $y$ ranges over $[b, b \vee x], z=x \wedge y$ ranges over the isomorphic interval $[b \wedge x, x]=[0, x]$, and $\mu(b, y)=\mu(0, z)$. Moreover $r(y)=r(b)+r(z)$. Therefore

$$
\begin{aligned}
P_{L}(\lambda) & =\sum_{b \wedge x=0} \sum_{z \in[0, x]} \mu(0, a) \mu(0, z) \lambda^{n-r(b)-r(z)} \\
& =\left[\sum_{z \in[0, x]} \mu(0, z) \lambda^{r(x)-r(z)}\right]\left[\sum_{b \wedge x=0} \mu(0, b) \lambda^{n-r(b)-r(x)}\right] \\
& =p_{x}(\lambda)\left[\sum_{b \wedge x=0} \mu(0, b) \lambda^{n-r(b)-r(x)}\right] \cdot \square
\end{aligned}
$$

\section{Examples}

As a special case of Theorem 2, suppose $x$ is modular copoint, and that exactly $\alpha$ points (atoms) $a$ of $L$ do not lie below $x$. Then these points $a$, together with 0 , are the 
only elements $b$ of $L$ satisfying $b \wedge x=0$. Moreover, $\mu(0, a)=-1, \mu(0,0)=1$, so

$$
p_{L}(\lambda)=p_{x}(\lambda)\left[\sum_{a}(-1)+\lambda\right]=p_{x}(\lambda)(\lambda-\alpha) .
$$

Thus if $L$ contains a maximal chain $0=x_{0}<x_{1}<\cdots<x_{n}=1$ such that each $x_{i-1}$ is modular in $\left[0, x_{i}\right]$ and such that exactly $\alpha_{i}$ atoms of $\left[0, x_{i}\right]$ do not lie below $x_{i-1}$, then $p_{L}(\lambda)=\left(\lambda-\alpha_{1}\right)\left(\lambda-\alpha_{2}\right) \ldots\left(\lambda-\alpha_{n}\right)$. It is easy to show that the condition that each $x_{i-1}$ is modular in $\left[0, x_{i}\right]$ is equivalent to the condition that each $x_{i}$ is modular in $L$. A class of geometric lattices with such a 'modular maximal chain' is the following: Let $\alpha_{1}, \alpha_{2}, \ldots, \alpha_{n}$ be any collection of positive integers with $\alpha_{n}=1$. Let $p_{1}, \ldots, p_{n}$ be $n$ independent points, and on the line $p_{i} p_{n}(1 \leqslant i \leqslant n-1)$ insert an additional $\alpha_{i}-1$ points. The geometric lattice $L$ of flats of this geometry contains a modular maximal chain, and $p_{L}(\lambda)=\left(\lambda-\alpha_{1}\right)\left(\lambda-\alpha_{2}\right) \ldots\left(\lambda-\alpha_{n}\right)$.

At this point it is natural to ask for a characterization of the modular elements of various geometric lattices. We state such a characterization when $L$ is the lattice of contractions of a finite graph. Recall that a contraction of a graph $G$ may be regarded as a partition $\pi$ of the vertices of $G$, such that the subgraph $H$ induced by each block $B$ of $\pi$ is connected [3, Chapter 6].

THEOREM 3. Let $L$ be the lattice of contractions of a doubly connected finite graph $G$. Then $\pi \in L$ is a modular element of $L$ if and only if the following conditions hold:

(i) At most one block $B$ of $\pi$ contains more than one vertex of $G$.

(ii) Let $H$ be the subgraph induced by the block $B$ of (i). Let $K$ be any connected component of the subgraph induced by $G-B$, and let $H_{1}$ be the graph induced by the set of vertices in $H$ which are connected to some vertex in $K$. Then $H_{1}$ is a clique (complete subgraph) of $G$.

The proof is of a routine nature and will be omitted.

If $G$ is not doubly connected, then the lattice of contractions of $G$ is a direct product of the lattices of contractions of the maximal doubly connected subgraphs of $G$, so Theorem 3 easily extends to arbitrary finite graphs $G$.

\section{REFERENCES}

[1] Garrett Birkhoff, Lattice Theory, Third Edition (American Mathematical Society, 1967).

[2] Henry Crapo, The Möbius Function of a Lattice, J. Combinatorial Theory 1 (1966), 126-131.

[3] Henry Crapo, and Gian-Carlo Rota, On the Foundations of Combinatorial Theory: Combinatorial Geometries, M.I.T. Press.

[4] Gian-Carlo Rota, On the Foundations of Combinatorial Theory, I: Theory of Möbius Functions, Z. Wahrscheinlichkeitstheorie 2 (1964), 340-368. 